\title{
Marking Xs, together and separately
}

\author{
Mary Ann Handel

\begin{abstract}
A chromosome imprinting mark is sensitive to meiotic pairing in the gamete of origin and identified through the 'histone code'.
\end{abstract}

Imprinting is an epigenetic modification that endows chromatin with a mark of parental origin $^{1}$. In mammals, specific chromosomal regions, and whole chromosomes, are marked as maternal or paternal. The nature of the mark is not fully understood, but both methylation of DNA and chromatin modifications have been implicated. This epigenetic mark can result in differences in transcriptional activity, which is how many imprinting effects are discovered. On page 100 of this issue, Bean et al. ${ }^{2}$ provide a new twist to the imprinting story. They find that in Caenorhabditis elegans, an unpaired X chromosome acquires a chromatin imprint during gametogenic meiosis.

\section{Singled out}

Nematode sex is unorthodox. XX individuals are hermaphrodites and produce both sperm and eggs, whereas XO individuals are committed males, producing only sperm. Previous work ${ }^{3,4}$ showed dimorphism in the behavior of the $\mathrm{X}$ chromosome in these two germ lines. In both germ lines, the $\mathrm{X}$ chromosome is inactive through most of meiotic prophase. But in XX individuals, the X chromosome accumulates activating histone modifications late in meiotic prophase during oogenesis, whereas the univalent, unpaired $\mathrm{X}$ chromosome in $\mathrm{XO}$ spermatocytes remains condensed and inactive.

Bean et al. ${ }^{2}$ asked if these modifications might predict differences between the maternal and paternal X chromosome in XX zygotes produced by mating XO males with XX individuals. They assayed accumulation on chromosomes of histone modifications associated with transcriptional activation-dimethylation of histone $\mathrm{H} 3$ at Lys4 and acetylation of H3. In early embryos they found an exceptional chromosome that did not have the histone modifications found on all other chromosomes until after several rounds of cell division. This chromosome was the paternally derived X chromosome. No such exceptional chromosome was found in the $\mathrm{XO}$ embryos,

Mary Ann Handel is at The Jackson Laboratory, 600 Main Street, Bar Harbor, Maine, USA and the University of Tennessee, Knoxville,

Tennessee, USA.e-mail: mahandel@jax.org

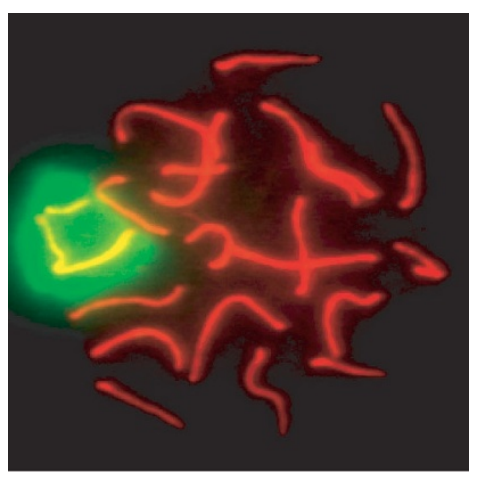

Figure 1 The paired $X$ and $Y$ chromosomes of the mammalian spermatocyte form a unique chromatin domain, marked here by immunostaining for phosphorylated histone H2AX (green), which is not found in the autosomal domain (red, immunostained for SYCP3, a protein of the synaptonemal complex).

whose sole $\mathrm{X}$ chromosome is maternally derived. They found an X chromosome without activating histone modifications in the sperm pronucleus of self-progeny, but it accumulates activating histones earlier than does the $\mathrm{X}$ chromosome derived from $\mathrm{XO}$ males.

What could this mean? Obviously, germline sex is important since the paternal $\mathrm{X}$ chromosome differs from the maternal X chromosome. But this is not the complete story, because the $\mathrm{X}$ chromosome from an $\mathrm{XO}$ male germ line has a more stable imprint than the paternal $\mathrm{X}$ chromosome from hermaphroditic (XX) male germ cells. So Bean et al. ${ }^{2}$ asked if the meiotic pairing status of the $\mathrm{X}$ chromosome might influence the imprint, with an unpaired X chromosome acquiring a more stable imprint. They used the C. elegans sex determination system and sex-reversing mutants to show that pairing status of the $\mathrm{X}$ chromosome, more than germline sex, determines the chromatin imprint. The establishment of the imprint is probably related to accumulation on the unpaired $\mathrm{X}$ chromosome (both in XO males and in mutant XO hermaphrodites undergoing oogenesis) of histone $\mathrm{H} 3$ methylated at Lys9 (H3-Lys9), a histone modification associated with the development of a repressive chromatin structure ${ }^{5}$.

\section{Silencing singles}

Meiotic silencing is not a new observation. It has been well documented in mammalian spermatocytes $^{6}$, where the $\mathrm{X}$ chromosome lacks a fully homologous pairing partner and together, the sex chromosomes assume a unique chromatin conformation (Fig. 1) known as the $\mathrm{XY}$ body that accumulates methylated H3-Lys9 (ref. 7) and many other proteins. What probably set the authors down their current path of research is the knowledge that transgene repeat arrays in C. elegans are silenced in the germ line and accumulate methylated H3-Lys9 (refs. 3,4). Meiotic silencing by unpaired DNA (MSUD) is best studied in Neurospora crassa $^{8}$, where genetic analysis has implicated an RNA interference-mediated silencing ${ }^{9}$. MSUD might be a mechanism of genomic defense or protection against invading sequences that has been hijacked by sex chromosomes in heterogametic species. But it could pose an inconvenience for the species if genes required for spermatogenesis resided on the X chromosome. In fact, the C. elegans X chromosome is depleted of spermatogenesis genes ${ }^{10}$ and silencing of the $\mathrm{X}$ chromosome in spermatogenesis may not be a problem.

But in mammals, there is a disproportionate abundance of spermatogenesis genes on the X chromosome ${ }^{11}$. So what gives? Many of these genes are expressed only before ${ }^{11}$ or after ${ }^{12}$ meiosis, circumventing the problem. But there are many housekeeping genes on the mammalian X chromosome, and another coping strategy is implied by the existence of autosomal paralogs that are expressed only in meiotic prophase spermatocytes ${ }^{6}$. Perhaps the $\mathrm{X}$ chromosome has not rid itself of this inconvenient silencing because it is important for the paternal $\mathrm{X}$ chromosome to be marked in the embryo. For instance, in XX marsupials and mammals, there is nonrandom inactivation of the paternal $\mathrm{X}$ chromosome globally or in the trophectoderm. Although the paternal $\mathrm{X}$ chromosome was previously thought to be active in the zygote, new evidence suggests that XX zygotes inherit a preinactivated paternal $\mathrm{X}$ chromosome $^{13}$. For XY heterogametic sexes, how better to acquire a paternal $\mathrm{X}$ chromosome mark than by recognition of lack of pairing partner? 
All this smacks of transvection and other homology-based silencing phenomena, which are increasingly recognized as important developmental mechanisms ${ }^{14}$. But the mark on the paternal X chromosome might not be indelible, because male mice who inherit their X chromosome from their fathers are not apparently developmentally impaired ${ }^{15}$. Notably, Bean et $a l .{ }^{2}$ found that the imprint of the C. elegans paternal X chromosome disappears after several rounds of mitosis as it accumulates histone modifications. Although the observed histone mark disappeared, we don't yet know if the underlying imprint also disappeared.

Now the questions begin: how is lack of pairing recognized, how is the imprint established and what is the underlying imprint? One thing is clear: the special status of the unpaired X chromosome of XO males is identified through the histone code. Although many will assume that the special X chromosome is transcriptionally silent, this has not yet been rigorously demonstrated. But whatever the epigenetic mark and whatever its function, knowledge of the roles of modified histones will take us closer to discovery.

This paper provides grist for the experimental mill of many of us: those interested in meiosis, reproduction, embryogenesis, transcriptional control, chromatin and evolution. It has wide implications to be tested in several systems, and I, for one, am quite eager to see where this takes us!

1. Reik, W. \& Walter, J. Nat. Rev. Genet. 2, 21-32 (2001).

2. Bean, C.J., Schaner, C.E. \& Kelly, W.G. Nat. Genet. 36, 100-105 (2004).
3. Kelly, W.G. et al. Development 129, 479-492 (2002).

4. Reuben, M. \& Lin, R. Dev. Biol. 245, 71-82 (2002).

5. Jenuwein, T. \& Allis, C.D. Science 293, 1074-1080 (2001).

6. McKee, B.D. \& Handel, M.A. Chromosoma 102, 71-80 (1993).

7. Peters, A.H.F.M. et al. Cell 107, 323-337 (2001).

8. Shui, P.K. \& Metzenberg, R.L. Genetics 161, 1483-1495 (2002)

9. Lee, D.W., Pratt, R.J., McLaughlin, M. \& Aramayo, R. Genetics 164, 821-828 (2003).

10. Reinke, V. et al. Mol. Cell 6, 605-616 (2000).

11. Wang, P.J., McCarrey, J.R., Yang, F. \& Page, D.C. Nat. Genet. 27, 422-426 (2001).

12. Moss, S.B., VanScoy, H. \& Gerton, G.L. Mamm. Genome. 8, 37-38 (1997).

13. Huynh, K.D. \& Lee, J.T. Nature advance online publication, 7 December 2003 (doi:10.1038/nature 02222).

14. Wu, C. \& Morris, J.R. Curr. Opin. Genet. Dev. 9 , 237-246 (1999).

15. Handel, M.A. \& Hunt, P.A. Mol. Reprod. Dev. 28, 337-340 (1991).

\title{
Diverse powerhouses
}

\section{David R Thorburn}

\begin{abstract}
Mitochondria in different tissues vary in number, morphology, ultrastructure, respiratory capacity and involvement in specific metabolic pathways. A comparison of the proteome of mitochondria from different tissues has identified the extent of the underlying variation in protein composition and how this may be determined by tissue-specific networks of coregulated genes.
\end{abstract}

For an organelle that once seemed to be loved only by hard core biophysicists, the mitochondrion has come a long way. Severe disorders of mitochondrial oxidative phosphorylation (OXPHOS) are now recognized as the most common group of inborn errors of metabolism, affecting at least 1 in 5,000 individuals ${ }^{1}$. Mitochondria are best known as the cell's energy source as producers of ATP. But, they also have pivotal roles in generating reactive oxygen species, calcium metabolism and cell death. Thus, it is not surprising that mitochondrial dysfunction contributes to diverse pathologies including neurodegeneration, diabetic complications and tumorigenesis. A recent study by Vamsi Mootha and colleagues ${ }^{2}$ in Cell provides a basis for connecting mitochondrial pathologies with molecular etiology by identifying new mitochondrial proteins and profiling the extent of their tissue-specific diversity.

David R Thorburn is at The Murdoch Childrens Research Institute and Genetic Health Services Victoria, Royal Children's Hospital, and Department of Paediatrics, University of Melbourne, Melbourne, Australia.

e-mail:david.thorburn@mcri.edu.au

\section{Compiling the list}

Over the past few years, different strategies have been used in attempting to determine the total number of proteins in mitochondria. These include epitope-tagging, systematic functional screening of whole-genome

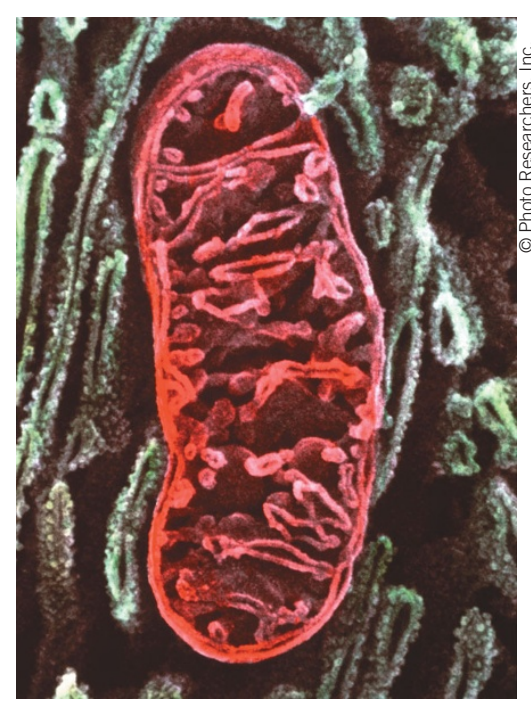

Figure 1 Colored high resolution scanning electron micrograph of a single mitochondrion in the cytoplasm of an intestinal epithelial cell. pools of mutants and proteomic analyses of highly purified mitochondria. Bioinformatic analyses have also been used to predict proteins with a classical mitochondrial targeting sequence or genes that are coregulated with genes encoding known mitochondrial proteins. These complementary approaches are necessary to overcome the various limitations in sensitivity and specificity of each method. Recent studies using such approaches in yeast are consistent with an estimate of 800-1,000 different mitochondrial proteins ${ }^{3-6}$.

Yeast mitochondria are relatively simple and uniform, but mammalian mitochondria vary widely between different tissues and often don't resemble the text-book version (Fig. 1) in morphology or composition ${ }^{7}$ (Table 1). The mitochondrial proteome of mammals is probably much larger and more diverse than that of yeast, but its size and variability have not been ascertained. Computational analysis predicts up to 4,000 mitochondrial proteins in humans ${ }^{3}$, which may be a true reflection of the complexity and diversity of our mitochondria or an overestimate caused by a systematic artifact. The first large-scale proteomic analysis of mammalian mitochondria, using human heart tissue, was 